\title{
Definitive tests for dengue fever: when and which should I use?
}

\author{
Hui Bin Yvonne Chan ${ }^{1}$, BSc, Choon How $\underline{H o w}^{1}$, MMed, FCFP, Chung Wai Mark $\underline{N g}^{2}$, MMed, FCFP
}

\begin{abstract}
Lisa, a 35-year-old woman, visited you for the first time, complaining about severe aches in her shoulders, hips and knee joints. She had also been feeling extremely tired for the past few days but reported no fever. You found out that her husband had been diagnosed with dengue a week before. In view of this history, you decided that it would be useful to conduct a definitive dengue diagnostic test.
\end{abstract}

\section{WHAT IS DENGUE FEVER?}

Dengue fever is an acute illness caused by an infection from one or more of the four serotypes of dengue viruses (DENV 1 to 4 ). The viruses are transmitted through the female mosquito species Aedes aegypti and, less commonly, Aedes albopictus. It is a dynamic, systemic disease with a wide clinical spectrum that includes both severe and non-severe clinical manifestations. Although the illness is usually self-limiting, for a minority of patients, dengue can cause life-threatening complications.

In 2009, the World Health Organization (WHO) introduced a revised scheme, which resulted in a paradigm shift in the approach to dengue fever. ${ }^{(1,2)}$ The proposed scheme emphasised the early recognition of warning signs, resulting in the optimisation of triage and management decisions. The following classifications were revised: dengue without warning signs; dengue with warning signs (abdominal pain, persistent vomiting, fluid accumulation, mucosal bleeding, lethargy, liver enlargement, increasing haematocrit with decreasing platelets); and severe dengue (dengue with severe plasma leakage, severe bleeding or organ failure). ${ }^{(3)}$

\section{HOW RELEVANT IS THIS TO MY PRACTICE?}

A total of 18,335, 11,286 and 13,115 dengue cases were recorded in Singapore in 2014, 2015 and 2016, respectively. ${ }^{(4)}$ In 2017, however, we have seen a sustained reduction of approximately two-thirds of dengue cases in the months leading up to 22 September (cumulative total of 2,066 reported cases). ${ }^{(5)}$ Project Wolbachia-Singapore is a new initiative that was started to combat dengue in the country. Male Aedes aegypti mosquitoes infected with Wolbachia (a naturally occurring bacteria) have been released in stages into the environment since October 2016. These male Wolbachia-carrying Aedes aegypti mosquitoes seek to mate with female Aedes aegypti, but the resulting eggs will not hatch (i.e. non-viable eggs) because the mating is biologically incompatible, thus leaving no offspring. ${ }^{(6)}$ The subsequent reduction in dengue cases suggests that such new vector control measures have been successful.

Although the number of dengue cases has been falling, countries in Southeast Asia (such as Thailand and Malaysia) and Latin America remain prime hotspots for the disease. ${ }^{(7)}$ With increasing air travel and greater mobility of both vector and human populations, the risk of imported dengue cases in Singapore is still high. ${ }^{(8)}$ Locally, other mosquito-borne viruses, such as Chikungunya and Zika, also often present with similar clinical features. Some common overlapping symptoms include fever, myalgia, maculopapular exanthema, retro-orbital pain and lymphadenopathy. ${ }^{(9,10)}$

Prompt and accurate diagnosis continues to be important. The lower prevalence of dengue, however, mandates a change in our clinical approaches to febrile illnesses and a review of the use of dengue diagnostic tests. The wide spectrum of febrile illnesses and atypical dengue presentations poses a real challenge to clinicians. ${ }^{(11)}$ Delayed diagnosis might lead to one or more of these unfortunate circumstances: (a) The patient will not be accorded the close vigilance required of dengue patients. (b) The patient will not be advised to monitor for danger signs. (c) The patient, who continues to be a reservoir of infection, will not be educated on vector bite prevention measures. (d) Potential dengue clusters may be missed due to a lack of epidemiological data. (e) Other unnecessary investigations, such as autoimmune markers or markers of myositis, may be ordered.

\section{WHAT CAN I DO IN MY PRACTICE?}

While dengue fever may be diagnosed clinically, laboratory tests can serve to confirm or secure a definitive diagnosis. Laboratory tests for dengue can be broadly categorised into definitive tests and nonspecific tests. Definitive tests include nonstructural protein 1 (NS1) antigen assay or dengue serology, while a complete blood count is an example of a nonspecific test. A 2011 study, which surveyed primary care physicians in Singapore, 
revealed that only about half of the physicians surveyed routinely ordered definitive tests for dengue; of these, three-quarters chose dengue serology testing. ${ }^{(12)}$

\section{Advantages of definitive tests}

Definitive tests for dengue, including rapid point-of-care tests, allow for early identification and prompt notification. They are especially useful in managing the clinical uncertainties often encountered in primary care. Having a confirmed diagnosis would also be helpful for patient education and encouraging compliance to follow-up checks. A confirmed diagnosis also allows physicians to better provide patients with appropriate advice, including steps for vector-bite prevention, thereby limiting transmission of the illness.

\section{When and which definitive test to use? Dengue NS1 antigen test}

In general, direct virus detection methods are used to obtain an early, definitive and serotype-specific identification, if it has been less than five days from the time of fever onset. NS1 antigen testing can be conducted fairly rapidly, and at a lower cost, than other methods such as viral isolation or viral reverse transcriptase-polymerase chain reaction. Ordering a dengue NS1 antigen assay within the first week of symptom onset is one of the initial investigations recommended by the Ministry of Health, Singapore, for dengue infections. ${ }^{(2)}$ NS1 antigen is glycoprotein that is synthesised by all flaviviruses and secreted from infected mammalian cells during the acute phase of infection. ${ }^{(13)}$ Most evaluations of the NS1 antigen assay mirror each other, indicating a moderately high sensitivity and very high specificity to dengue infection. ${ }^{(14,15)}$ There is also evidence that the sensitivity of NS1 antigen detection is higher in primary infection (>90\%) than in secondary infection $(60 \%-80 \%) .{ }^{(16)}$ High levels of NS1 antigen were also found to be correlated with higher levels of viraemia and, thus, could potentially be a predictor for severe illness. ${ }^{(16-19)}$

\section{Dengue IgM/IgG serological test}

Immunoglobulin M (IgM) enzyme-linked immunosorbent assay (ELISA) and/or immunoglobulin G (IgG) ELISA are the tests of choice after the first five days of illness. In the later stages of illness, dengue serology is more useful, as NS1 antigen remains detectable for up to only nine days and the patient may thus test negative for dengue. ${ }^{(20)}$ Dengue-specific IgM is usually expressed by Day 5 of illness onset and is present in higher levels than IgG in primary infection. ${ }^{(1,21)}$ Distinguishing between primary and secondary dengue is often not done in the primary care setting, as it may not be of practical relevance.

\section{Available practical options}

Physicians in both public and private practice can tap on laboratories in restructured hospitals or private laboratories for definitive dengue tests. While a patient's blood samples can be sent to the Environmental Health Institute laboratory at the National Environment Agency, the laboratory currently offers definitive testing only for the purpose of epidemiological surveillance.
Commercially available rapid point-of-care test kits, such as the Standard Diagnostics (SD) BIOLINE Dengue Duo kit (Standard Diagnostics Inc, Gyeonggi-do, Republic of Korea) are often used in the primary care setting. The SD BIOLINE Dengue Duo test is a one-step assay to detect dengue NS1 antigen and IgM/lgG in human serum, plasma or whole blood, and can be completed within 15-20 minutes. In 2014, a prospective cohort study conducted in Singapore found that this test has an overall sensitivity and specificity of $93.9 \%$ (95\% confidence interval $[\mathrm{Cl}]$ 88.8\%-96.8\%) and 92.0\% (95\% Cl 81.2\%-96.9\%), respectively. ${ }^{(22)}$ Another locally available rapid test kit is the MP Diagnostics MULTISURE Dengue Ab/Ag test kit (MP Biomedicals, LLC, Santa Ana, CA, USA). These commercially available test kits, which could serve as alternative options in primary care, can enhance dengue diagnostics in an endemic setting.

\section{TAKE HOME MESSAGES}

1. Dengue is a dynamic, systemic disease with a wide clinical spectrum that includes both severe and non-severe clinical manifestations.

2. Prompt diagnosis and notification are crucial for initiating early vector control measures and early management, especially in atypical cases or when presented with a differential diagnosis.

3. In general, laboratory tests for dengue can be broadly categorised into nonspecific tests (e.g. complete blood count) and definitive tests (e.g. NS1 antigen test, dengue serology).

4. Definitive tests are useful for confirmation, as symptoms of early dengue overlap with those of many other febrile illnesses.

5. Dengue diagnostic results provide important epidemiological data and are crucial to initiate early vector control measures in the community.

6. Having a confirmed diagnosis allows the primary care physician to provide appropriate follow-up and patient education.

7. The NS1 antigen assay has a moderately high sensitivity and very high specificity to dengue infection.

8. NS1 antigen can be detected early in the course of illness, while positive serology is observed later on.

9. Primary care physicians have numerous laboratory options for definitive tests for dengue, including commercially available point-of-care test kits that incorporate both dengue NS1 antigen testing and serology.

The point-of-care Dengue Duo diagnostic kit showed a positive diagnosis of dengue infection. You assured Lisa that her symptoms can be safely attributed to dengue, and that her illness was an atypical presentation. You promptly notified the National Environment Agency of the dengue case, which helped to trigger vector control measures in Lisa's neighbourhood. 
ABSTRACT Dengue is a mosquito-borne viral disease that has established itself globally in both endemic and epidemic transmission cycles. While diagnostic decision-making for dengue is often guided by clinical judgement, definitive laboratory tests, including rapid point-of-care tests, have many advantages in the primary care setting. These include providing epidemiological data and diagnostic clarity for atypical cases, as well as contributing to patient education and compliance. This article discussed the types of diagnostic methods for dengue, when to use them and the appropriateness of each test. Viral detection diagnostic methods such as NS1 antigen assays are generally used within the first week of illness onset, whereas dengue serology testing is most appropriate after that time frame. Locally available rapid point-of-care tests, which include both assays in one convenient test kit, can enhance dengue diagnosis in an endemic setting.

Keywords: definitive test, dengue, diagnostic tests, primary care, test kits

\section{ACKNOWLEDGEMENTS}

We would like to thank Dr Jack Tan, Medical Director, Northeast Medical Group, for sharing on his clinical practice with the authors.

\section{REFERENCES}

1. World Health Organization, Special Programme for Research and Training in Tropical Diseases. Dengue guidelines for diagnosis, treatment, prevention and control: new edition [online]. Available at: http://apps.who.int/iris/ bitstream/10665/44188/1/9789241547871_eng.pdf. Accessed October 12 2017

2. Ng CW. Revisiting the approach to dengue: the primary care perspective. Singapore Fam Physician 2015; 41:65-73.

3. World Health Organization. Handbook for Clinical Management of Dengue [online]. Available at: http://www.wpro.who.int/mvp/documents/handbook for_clinical_management_of_dengue.pdf. Accessed October 12, 2017.

4. National Environment Agency. Dengue surveillance data, Oct-Dec 2016 [online]. Available at: http://www.nea.gov.sg/docs/default-source/public-health/ Dengue/q4-2016-dengue-surveillance-data.pdf. Accessed October 12, 2017.

5. National Environment Agency. Dengue Cases [online]. Available at: http://www. nea.gov.sg/public-health/dengue/dengue-cases. Accessed September 24, 2017.

6. National Environmental Agency. Wolbachia-Aedes Mosquito Suppression Strategy [online]. Available at: http://www.nea.gov.sg/public-health/ environmental-public-health-research/wolbachia-technology. Accessed September 25, 2017.

7. Bhatt S, Gething PW, Brady OJ, et al. The global distribution and burden of dengue. Nature 2013: 496:504-7.

8. Murray NE, Quam MB, Wilder-Smith A. Epidemiology of dengue: past, present and future prospects. Clin Epidemiol 2013; 5:299-309.

9. Beltrán-Silva SL, Chacón-Hernández SS, Moreno-Palacios E, Pereyra-Molina JA. Clinical and differential diagnosis: dengue, chikungunya and Zika. Rev Médica del Hosp Gen México 2016 Oct 19.

10. loos S, Mallet HP, Leparc Goffart I, et al. Current Zika virus epidemiology and recent epidemics. Med Mal Infect 2014; 44:302-7.

11. Wiwanitkit S, Wiwanitkit V. Afebrile dengue: an easily forgotten manifestation. Ann Trop Med Public Heal 2013; 6:686.

12. Lee $L K$, Thein TL, Kurukularatne $C$, et al. Dengue knowledge, attitudes, and practices among primary care physicians in Singapore. Ann Acad Med Singapore 2011; 40:533-8.

13. Peeling RW, Artsob H, Pelegrino JL, et al. Evaluation of diagnostic tests: dengue. Nat Rev Microbiol 2010; 8(12 Suppl):S30-8.

14. Solanke VN, Karmarkar MG, Mehta PR. Early dengue diagnosis: role of rapid NS1 antigen, NS1 early ELISA, and PCR assay. Trop J Med Res 2015; 18:95-9.

15. Chaterji S, Allen JC Jr, Chow A, Leo YS, Ooi EE. Evaluation of the NS1 rapid test and the WHO dengue classification schemes for use as bedside diagnosis of acute dengue fever in adults. Am J Trop Med Hyg 2011; 84:224-8.

16. Hang VT, Nguyet NM, Trung DT, et al. Diagnostic accuracy of NS1 ELISA and lateral flow rapid tests for dengue sensitivity, specificity and relationship to viraemia and antibody responses. PLoS Negl Trop Dis 2009; 3:e360.

17. Libraty DH, Young PR, Pickering D, et al. High circulating levels of the dengue virus nonstructural protein NS1 early in dengue illness correlate with the development of dengue hemorrhagic fever. J Infect Dis 2002; 186:1165-8.

18. Avirutnan P, Punyadee N, Noisakran S, et al. Vascular leakage in severe dengue virus infections: a potential role for the nonstructural viral protein NS1 and complement. J Infect Dis 2006; 193:1078-88.

19. Libraty DH, Endy TP, Houng HS, et al. Differing influences of virus burden and immune activation on disease severity in secondary dengue-3 virus infections. J Infect Dis 2002; 185:1213-21.

20. Kumarasamy V, Wahab AH, Chua SK, et al. Evaluation of a commercial dengue NS1 antigen-capture ELISA for laboratory diagnosis of acute dengue virus infection. J Virol Methods 2007; 140:75-9.

21. Shu PY, Huang JH. Current advances in dengue diagnosis. Clin Diagn Lab Immunol 2004; 11:642-50.

22. Gan VC, Tan LK, Lye DC, et al. Diagnosing dengue at the point-of-care: utility of a rapid combined diagnostic kit in Singapore. PLoS One 2014; 9:e90037. 


\section{SINGAPORE MEDICAL COUNCIL CATEGORY 3B CME PROGRAMME} (Code SMJ 201711A)

1. Dengue fever is an acute illness caused by infection from one or more of the four serotypes of dengue viruses (DENV 1 to 4 ).

2. In 2009, the World Health Organization (WHO) introduced a revised scheme emphasising the early recognition of warning signs to optimise triage and management decisions.

3. There has been a sustained increase in the number of dengue cases in Singapore since 2017.

4. A lower prevalence of dengue will mandate a change in clinical approaches to febrile illnesses and a review of the use of dengue diagnostic tests.

5. While dengue may be clinically diagnosed, laboratory tests are helpful to confirm or secure a definitive diagnosis.

6. Nonspecific dengue laboratory tests include nonstructural protein 1 (NS1) antigen assays and dengue serology tests.

7. A complete blood count is an example of a definitive dengue laboratory test.

8. Dengue diagnostic results do not provide epidemiological data and are therefore not necessary to spark off early vector control measures in the community.

9. Having a confirmed diagnosis of dengue allows the primary care physician to provide appropriate follow-up and patient education.

10. Definitive tests are useful for confirmation of dengue, as the symptoms of early dengue overlap with those of many other febrile illnesses.

11. In general, direct virus detection methods, such as NS1 antigen testing, are used later in the course of illness.

12. NS1 antigen is glycoprotein that is synthesised by all flaviviruses and secreted from infected mammalian cells during the acute phase of infection.

13. The NS1 antigen assay has both very high sensitivity and specificity to dengue infection.

14. High levels of NS1 antigen can potentially be a predictor of severe illness.

15. In the later stages of illness, dengue serology is more useful, as NS1 antigen remains detectable for up to only nine days and may thus return negative results for dengue.

16. Dengue specific immunoglobulin M (IgM) is usually expressed at lower levels than immunoglobulin G (lgG) in primary infection.

17. Primary care physicians do not have available laboratory options for definitive tests for dengue.

18. A patient's blood samples can be sent to the Environmental Health Institute laboratory at the National Environment Agency for diagnostic purposes.

19. Rapid point-of-care test kits incorporate both dengue NS1 antigen and IgM/lgG assays.

20. There are currently no commercially available rapid point-of-care test kits in Singapore.

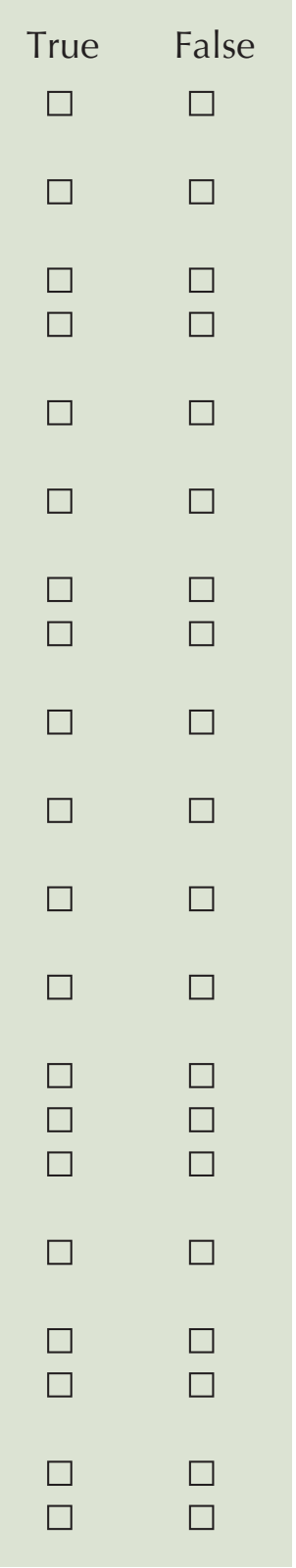

\section{Doctor's particulars:}

Name in full:

MCR no:

Specialty:

Email:

\section{SUBMISSION INSTRUCTIONS:}

Visit the SMJ website: http://www.smj.org.sg/current-issue and select the appropriate quiz. You will be redirected to the SMA login page.

For SMA member: (1) Log in with your username and password (if you do not know your password, please click on 'Forgot your password?'). (2) Select your answers for each quiz and click 'Submit'.

For non-SMA member: (1) Create an SMJ CME account, or login with your SMJ CME username and password (for returning users). (2) Make payment of SGD 21.40 (inclusive of $7 \%$ GST) via PayPal to access this month's quizzes. (3) Select your answers for each quiz and click 'Submit'.

\section{RESULTS:}

(1) Answers will be published online in the SMJ January 2018 issue. (2) The MCR numbers of successful candidates will be posted online at the SMJ website by 3 January 2018. (3) Passing mark is $60 \%$. No mark will be deducted for incorrect answers. (4) The SMJ editorial office will submit the list of successful candidates to the Singapore Medical Council. (5) One CME point is awarded for successful candidates.

Deadline for submission: (November 2017 SMJ 3B CME programme): 12 noon, 27 December 2017. 